\title{
Pungency and fruit quality in Mexican landraces of piquín pepper (Capsicum annuum var. glabriusculum) as affected by plant growth environment and postharvest handling
}

\author{
Deisy D. Díaz-Sánchez ${ }^{1}$, Pedro A. López ${ }^{2}$, Higinio López-Sánchez ${ }^{2}$, Hilda V. Silva-Rojas ${ }^{1}$, \\ Alfonso A. Gardea-Béjar ${ }^{3}$, Nicacio Cruz-Huerta ${ }^{1}$, Iván Ramírez-Ramírez ${ }^{1}$, \\ and Víctor A. González-Hernández ${ }^{1 *}$
}

${ }^{1}$ Colegio de Postgraduados, Campus Montecillo, Carr. México-Texcoco km 36.5, Montecillo 56230, Texcoco, Estado de México, México. *Corresponding author (vagh@colpos.mx).

${ }^{2}$ Colegio de Postgraduados, Campus Puebla, Carr. Federal México-Puebla km 125.5, Santiago Momoxpan 72760, Puebla, México. ${ }^{3}$ Centro de Investigación y Desarrollo, A.C. (CIAD), Carr. Gustavo E. Astiazarán Rosas N 46, CP 83304, Hermosillo, Sonora, México.

Received: 6 March 2021; Accepted: 27 July 2021; doi:10.4067/S0718-58392021000400546

\begin{abstract}
The variability in fruit pungency, size and quality was analyzed in 31 landraces of piquín pepper (Capsicum апnиит L. var. glabriusculum (Dunal) Heiser \& Pickersgill) collected from 10 Mexican states. The response of fruits collected in situ and harvested in greenhouse was compared. Pungency was estimated by measuring capsaicinoids content with HPLC. Additionally, pungency was recorded along fruit maturity stages in greenhouse-grown fruits. On average, greenhouseproduced fruits were markedly more pungent than fruit collected from field locations (29485 vs. 6114 SHU). Fieldcollected fruits averaged $5017 \mu \mathrm{g} \mathrm{mL}^{-1}$ capsaicin content, while dihydrocapsaicin averaged $7618 \mu \mathrm{g} \mathrm{mL}^{-1}$. In contrast, greenhouse-harvested fruits contained substantially more capsaicin and dihydrocapsaicin (34762 and $26174 \mu \mathrm{g} \mathrm{mL} \mathrm{L}^{-1}$, respectively), while the capsaicin:dihydrocapsaicin ratio inverted. The three most pungent field-collected landraces averaged 35\% of the pungency measured in the three most pungent greenhouse-grown fruits. Pungency increased as maturity stage advanced: green (14813 SHU) < intermediate (24767 SHU) < red (29485 SHU). The fruits collected from 31 field locations had lower titratable acidity, higher content of total soluble solids, and higher maturity index than fruits harvested in greenhouse. Greenhouse harvested fruits were larger and heavier than those collected in the field.
\end{abstract}

Key words: Capsaicinoids, fruit quality, fruit size, landraces, maturity stages, piquín pepper, pungency.

\section{INTRODUCTION}

Piquín, chiltepín or bird pepper (Capsicum annиum L. var. glabriusculum (Dunal) Heiser \& Pickersgill) (Cag) is one of the preferred peppers in Mexico (Bañuelos et al., 2008). The growing zone for this pepper spans from southern USA to South America (Hernández-Verdugo et al., 2012). Cag is considered the wild ancestor of numerous cultivated peppers of the C. аппиит species (Montoya-Ballesteros et al., 2010), such as jalapeño, serrano, ancho, pasilla, guajillo, árbol, and other commercial peppers. Cag fruits are frequently harvested from wild plants by uprooting the whole plant without being replanted (Bañuelos et al., 2008). Consequently, the in situ conservation of this plant genetic resource is at risk. Yet, commercial Cag plantings are scarce due mainly to the difficulty of germinating the seeds (Villalón et al., 2013; CanoVázquez et al., 2015) and to the lack of genetically bred commercial varieties. On the other hand, the Cag fruits harvested from plantations may show different changes compared to wild-harvested fruits (Sandoval-Rangel et al., 2018). 
Cag fruit is small, turns red when ripe, and is often highly pungent. It is up to 40 times more pungent than popular peppers such as serrano and jalapeño, and it is consumed fresh or dried for food seasoning (Quintero Barrera, 2000). According to Chasing-Chilli (2020) the piquín/chiltepín pungency ranges between 50000 and 100000 SHU (Scoville heat units). However, González-Zamora et al. (2015) reported pungency levels from 324924 to 1765283 SHU for piquín fruits at the green maturity stage harvested from plants cultivated in irrigated field conditions in northern México. Despite its high pungency, Cag is regarded as having a pleasant taste, and its hot sensation presumably fades quickly without irritating the stomach (Martínez et al., 2006; Bañuelos et al., 2008). Most studies on piquín pepper have focused on describing fruit morphological and genetic variability from wild local landraces (Hernández-Verdugo et al., 2001; Alonso et al., 2012; Narez-Jiménez et al., 2014; Murillo-Amador et al., 2015). However, fruit quality traits such as antioxidant content and taste, have been measured only in a few regional landraces (also called accessions, ecotypes, or morphotypes) (Montoya-Ballesteros et al., 2010; Rodríguez-Maturino et al., 2012; Flores-González et al., 2018). Other quality traits like fruit size, color, and flavor have scarcely been reported for piquín pepper, contrasting with the vast information available for the commercially cultivated chilis. For example, for nine wild piquín pepper landraces from northeast México (Moreno-Ramírez et al., 2018), the reported pungency ranged from 22190 to 44035 SHU. Phenolic compounds content and antioxidant capacities varied too. The changes in piquín pepper pungency when used for elaborating sauces and pickles, has also been reported (Montoya-Ballesteros et al., 2010).

Capsaicinoids synthesized in the placenta of Capsicum spp. fruits are responsible for pungency (Barchenger and Bosland, 2016). Barchenger and Bosland (2016) reported 20 different capsaicinoids in C. annuum, and two of them, capsaicin and dihydrocapsaicin, account for about $90 \%$ of the total capsaicinoid content in chili peppers (de Aguiar et al., 2016).

In this study, we explored the variation of piquín accessions in a wide sample of 31 Mexican landraces collected across 10 Mexican states, by measuring their capsaicinoids content, titratable acidity, total soluble solids content, and the palatability index. These traits were assessed both on fruits obtained from collected samples at different 31 sites and conditions, and on fruits harvested from plants of the same landraces grown under a greenhouse and supplied with water and nutrients with a drip irrigation system.

\section{MATERIALS AND METHODS}

\section{Plant material and experimental management}

The 31 piquín or bird pepper (Capsicum annuum L. var. glabriusculum [Dunal] Heiser \& Pickersgill,) (Cag) fruit accessions were obtained from 10 Mexican states and had four different provenances: harvested from backyard plots, harvested from commercial field plots, harvested from wild plants, or bought in local markets (Table 1). Pungency and fruit quality was assessed in the collected fruits, as well as in fruits harvested from plants grown in a greenhouse fitted with a drip irrigation system at Texcoco (19 $23^{\prime} 40^{\prime \prime} \mathrm{N}, 99^{\circ} 01^{\prime} 45^{\prime \prime} \mathrm{W} ; 2250 \mathrm{~m}$ a.s.l.) In the greenhouse temperatures varied across the year from 8.9 to $26.3^{\circ} \mathrm{C}$, with a mean value of $16.3^{\circ} \mathrm{C}$. These values are very similar to State of México field conditions where mean annual temperature is $16^{\circ} \mathrm{C}$. Plants were grown from seeds extracted from collected fruits and germinated in polystyrene trays of 200 cavities filled with a 1:1 mixture of agrolite and peatmoss as substrate (Cano-Vázquez et al., 2015), under greenhouse conditions. When seedlings reached $7 \mathrm{~cm}$ tall, they were transplanted into $10 \mathrm{~L}$ black bags filled with volcanic, porous red-gravel, 2-4 mm diameter (locally called 'tezontle'), commonly used as substrate for hydroponic cropping. Nutrients were supplied with a Steiner solution (Steiner, 1961) provided nine times a day for 5 min periods.

The greenhouse piquín plants were randomly distributed in two pot lines, $1 \mathrm{~m}$ apart, and individual plants were $40 \mathrm{~cm}$ apart along each line. When plants were between 14 and 18 mo-old (after transplant), fruits were harvested at three stages of maturity, according to the BBCH scale proposed by Feldmann and Rutikanga (2021) for chili fruits: green (growth stage 703), intermediate (beginning to acquire the red color, considered commercial harvest maturity; growth stage 801), and mature red (growth stage 809). The pre- and postharvest handling were identical for all landraces. 
Table 1. Geographic locations of the 31 piquín pepper Mexican landraces evaluated in this study.

\begin{tabular}{|c|c|c|c|c|c|c|}
\hline ID & Landrace & Latitude & Longitude & State & Location (city/town) & Origin \\
\hline 1 & Ags01 & 22.145 & -102.273 & Aguascalientes & Aguascalientes & Local market \\
\hline 2 & $\mathrm{Ags} 02$ & 22.887 & -102.291 & Aguascalientes & CONAGUA & Backyard plot \\
\hline 3 & $\mathrm{Ags03}$ & 22.865 & -102.289 & Aguascalientes & Aguascalientes & Cultivated plot \\
\hline 4 & Dur01 & 24.027 & -104.653 & Durango & Durango & Local market \\
\hline 5 & Hgo01 & 20.970 & -98.508 & Hidalgo & Tlamamala & Wild plants \\
\hline 6 & Mich01 & 20.336 & -102.022 & Michoacán & La Piedad & Wild plants \\
\hline 7 & Mich02 & 20.336 & -102.022 & Michoacán & Michoacán & Backyard plot \\
\hline 8 & Nay02 & 21.726 & -105.058 & Nayarit & El Jicote & Wild plants \\
\hline 9 & Oax01 & 17.332 & -95.418 & Oaxaca & San José de las Flores & Backyard plot \\
\hline 10 & Oax03 & 16.948 & -96.751 & Oaxaca & Zaachila & Backyard plot \\
\hline 11 & Oax05 & 16.948 & -96.751 & Oaxaca & Zaachila & Backyard plot \\
\hline 12 & Oax07 & 16.612 & -96.852 & Oaxaca & San Martín Lachila & Local market \\
\hline 13 & Oax10 & 16.612 & -96.852 & Oaxaca & San Martín Lachila & Backyard plot \\
\hline 14 & Pue01 & 20.284 & -97.964 & Puebla & Xicotepec & Wild plants \\
\hline 15 & Pue04 & 20.018 & -97.523 & Puebla & Cuetzalan & Cultivated plot \\
\hline 16 & Qro01 & 20.907 & -99.933 & Querétaro & Tolimán Xiti & Wild plants \\
\hline 17 & Qro02 & 20.689 & -99.820 & Querétaro & Higuerillas & Wild plants \\
\hline 18 & Qro03 & 20.907 & -99.933 & Querétaro & El Patol & Wild plants \\
\hline 19 & Qro04 & 20.778 & -99.872 & Querétaro & El Patol & Wild plants \\
\hline 20 & Qro06 & 20.907 & -99.933 & Querétaro & San Antonio Tolimán & Wild plants \\
\hline 21 & Qro07 & 21.219 & -99.471 & Querétaro & Jalpan de Serra & Wild plants \\
\hline 22 & Qro09 & 20.786 & -100.050 & Querétaro & Colón & Wild plants \\
\hline 23 & Qro10 & 21.219 & -99.471 & Querétaro & Jalpan de Serra & Wild plants \\
\hline 24 & Sin05 & 28.681 & -110.455 & Sinaloa & El Porvenir & Wild plants \\
\hline 25 & Ver01 & 20.979 & -98.175 & Veracruz & Chicontepec & Local market \\
\hline 26 & Ver04 & 20.702 & -97.310 & Veracruz & Cerro Verde & Cultivated plot \\
\hline 27 & Ver06 & 20.444 & -97.325 & Veracruz & Papantla & Cultivated plot \\
\hline 28 & Ver08 & 20.956 & -97.406 & Veracruz & Tuxpan & Cultivated plot \\
\hline 29 & Ver09 & 20.956 & -97.406 & Veracruz & Tuxpan & Local market \\
\hline 30 & Ver11 & 20.702 & -97.310 & Veracruz & Cazones & Local market \\
\hline 31 & Ver12 & 20.672 & -97.206 & Veracruz & Jilotepec & Wild plants \\
\hline
\end{tabular}

\section{Capsaicinoids extraction}

Four $10 \mathrm{~g}$ fruit samples (fresh weight) were taken from each landrace, both from field-collected and greenhouse-harvested, washed with distilled water, and freeze-dried (benchtop freeze dryer FreeZone 4.5 L, Labconco, Kansas City, Missouri, USA) for $3 \mathrm{~d}$ to preserve their integrity. The dried samples were ground in a $1 \mathrm{~mm}$ sieve mill (Thomas Model 4 Wiley mill, Thomas Scientific, Swedesboro, New Jersey, USA) to a fine powder. From each sample, $0.5 \mathrm{~g}$ powder were mixed with 5 $\mathrm{mL}$ acetonitrile into a $15 \mathrm{~mL}$ plastic centrifuge tube, and placed in a water bath for $5 \mathrm{~h}$ at $60^{\circ} \mathrm{C}$. Every hour the tubes were stirred vigorously for $1 \mathrm{~min}$. Thereafter, the tubes were cooled to room temperature in the dark. In every tube, evaporated acetonitrile was replaced to $5 \mathrm{~mL}$. Each resulting extract was filtered with polytetrafluoroethylene (PTFE) syringe filters (25 mm diameter, $0.45 \mu \mathrm{m}$ pore) and stored at $4{ }^{\circ} \mathrm{C}$ until HPLC analysis.

\section{Capsaicinoid quantification by HPLC}

Capsaicin (C) and dihydrocapsaicin (D) contents were determined in $20 \mu \mathrm{L}$ of each liquid extract. Samples were injected into an HPLC 1260 equipped with a DAD detector (Agilent, Santa Clara, California, USA). The running conditions were: Hypersil ODS MR column $(25 \mathrm{~cm}, 4.6 \mathrm{~mm}, 5 \mu \mathrm{M})$, a mobile phase of a 45:55 acetonitrile-water mixture, flow of $1.5 \mathrm{~mL} \mathrm{~min}^{-1}$ and $20 \mathrm{~min}$ running time per injection. For automatic detection and quantification, calibration curves $\left(\mathrm{R}^{2}=0.99\right)$ were constructed with external standards of capsaicin (8-methyl- $n$-vanillyl-6-nonenamide) and dihydrocapsaicin (8-methyl- $n$-vanillyl-nonanamide) (Sigma-Aldrich, St. Louis, Missouri, USA) at 0.25, 0.5 and 1.0 $\mathrm{mg} \mathrm{mL}^{-1}$ concentrations. In greenhouse-harvested fruits, capsaicinoid contents were measured at three different maturity fruit stages: green, intermediate, and red. 
Peak areas obtained by HPLC were transformed to Scoville heat units (SHU) using the equations proposed by the AOAC Official Method 995.03 (AOAC, 1995):

$$
\begin{aligned}
& \mathrm{C}=(\mathrm{Pc} / \mathrm{Ps}) \times(\mathrm{Cs} / \mathrm{Wt}) \times(10 / 0.89) \times 16100 \\
& \mathrm{D}=(\mathrm{Pd} / \mathrm{Ps}) \times(\mathrm{Cs} / \mathrm{Wt}) \times(10 / 0.93) \times 16100
\end{aligned}
$$

where $\mathrm{C}$ is capsaicin content (SHU); D is dihydrocapsaicin content (SHU); Pc and Pd are peak areas for capsaicin and dihydrocapsaicin, respectively; Ps is peak area of the corresponding standard; Cs is concentration of the standard solution $\left(\mathrm{mg} \mathrm{mL}^{-1}\right)$; Wt is sample weight $(\mathrm{g})$. Conversion to $\mathrm{SHU}$ from $\mathrm{mg} \mathrm{mL}^{-1}$ was calculated with the formula $1 \mu \mathrm{g}$ capsaicinoids $\mathrm{g}^{-1}$ dry weight $=15$ SHU (Wall et al., 2001).

\section{Fruit size and quality traits}

Fruit length and width (mm) were measured with a digital caliper (Truper, Mexico) in 20 randomly selected fruits per accession. Fruit dry-weight (mg) was measured with an analytical balance (Sartorius Handy H51, Sartorius, Gottingen, Germany) in the 20 previously selected fruits after they were oven dried to constant weight. The roundness index per fruit was calculated as the length to width ratio. The Manual of Descriptors for Capsicum spp. (IPGRI, AVRDC, CATIE, 1995) was used as a guide for these measurements.

Titratable acidity (TA), as percentage of citric acid was determined in a sample of $5 \mathrm{~g}$, according to the AOAC technique (AOAC, 1995). The fruit $\mathrm{pH}$ was measured with a digital pH meter (HI 2211, Hanna Instruments, Leighton Buzzard, UK). Total soluble solids (TSS, in ${ }^{\circ}$ Brix) were measured in a drop of juice obtained by fruit maceration, with a digital refractometer (PR-100, ATAGO, Tokyo, Japan). For each quality characteristic, three samples were measured, and the results averaged. The maturity index (MI) was calculated as the ratio MI $=$ TSS/TA.

\section{Experimental design and statistical analyses}

Both for the field-collected fruits and for the greenhouse-harvested fruits, the experimental treatments were the 31 landraces randomly distributed under a completely randomized design with four replicates (4 plants). Capsaicin and dihydrocapsaicin contents, as well as total capsaicinoid content (Total = capsaicin + dihydrocapsaicin), were analyzed under a completely randomized design with three replicates. All data were statistically analyzed with ANOVA, and treatment means were ranked by multiple mean comparisons with the Tukey's test $(\mathrm{P} \leq 0.05)$ when needed, with InfoStat (Grupo InfoStat, FCA, Universidad Nacional de Córdoba, Córdoba, Argentina). Based on the statistical analyses of morphological and quality fruit traits, the most significant variables were fed into a principal component analysis data array in SAS 9.4 (SAS Institute, Cary, North Carolina, USA). Landraces were classified into similarity groups with a cluster analysis by using Minitab v18 (Minitab, State College, Pennsylvania, USA).

\section{RESULTS AND DISCUSION}

\section{Capsaicinoid content as affected by growth environment and postharvest handling}

The average total capsaicinoid content in field collected fruits from the 31 landraces was 6114 SHU. This content split into $40 \%$ for capsaicin content and 60\% for dihydrocapsaicin content (Figure 1). Fruits harvested in the greenhouse had 29485 SHU, of which capsaicin accounted for $57 \%$ while dihydrocapsaicin was the remaining $43 \%$. The greenhouse fruits were almost five times more pungent $(\mathrm{P}<0.05)$ than the field-collected fruits.

\section{Pungency variation in field-collected fruits}

The average content of total capsaicinoids for field-collected fruits was $408 \mu \mathrm{g} \mathrm{mL} \mathrm{L}^{-1}$ for the 31 landraces (Table 2). Dihydrocapsaicin content was higher than capsaicin content (246vs. $162 \mu \mathrm{g} \mathrm{mL}^{-1}$; i.e., $60 \%$ vs. 40\%). Pungency varied greatly among the evaluated landraces, from low (2101 SHU) to high pungency (20693 SHU), thus spanning a wide range of consumer preferences. These differences result from the combination of inherent genetic differences among landraces, and to the effects of multiple environmental factors associated with climate, soil, and postharvest handling. The environmental variation is associated to the contrasting soil and climate conditions inherent to the 10 geographic studied Mexican regions. Furthermore, different planting settings (i.e., wild plants, plants from commercial field plots, plants cultivated in small backyard plots), as well as unidentified differences in postharvest handling, most particularly 
from market-bought fruits, may have influenced the observed variability. Harvell and Bosland (1997) demonstrated that pungency was more affected by environment than by genotype, in several chili genotypes. Similarly, González-Zamora et al. (2013) reported that high temperatures above $40{ }^{\circ} \mathrm{C}$ interact significantly with genotype since hot weather increased pungency in some Capsicum species while in others it became decreased.

Figure 1. Average contents of capsaicin, dihydrocapsaicin, and total capsaicinoids in fruits from 31 field locations vs. greenhouse harvested plants. Total capsaicinoids content is the sum of capsaicin and dihydrocapsaicin contents.
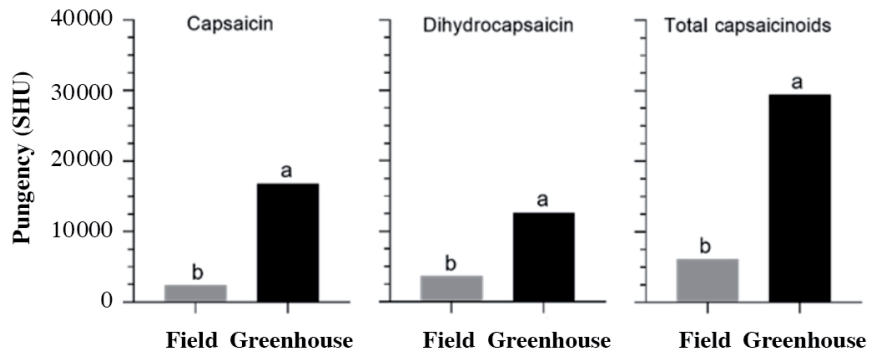

Values with different letters are significantly different (Tukey, $\mathrm{p}<0.05$ ).

Table 2. Capsaicinoid content in fruits of $\mathbf{3 1}$ piquín pepper landraces as they were sampled at different field locations in México.

\begin{tabular}{|c|c|c|c|c|}
\hline Landrace & Capsaicin & Dihydrocapsaicin & Total capsaicinoids & Pungency \\
\hline & $\mu \mathrm{g} \mathrm{mL^{-1 }}$ & $\mu \mathrm{g} \mathrm{mL}^{-1}$ & $\mu \mathrm{g} \mathrm{mL} L^{-1}$ & SHU \\
\hline Ags01 & $118 \pm 43 \mathrm{~cd}$ & $132 \pm 42 \mathrm{~cd}$ & $250 \pm 85 \mathrm{~cd}$ & $3748 \pm 1273 \mathrm{~cd}$ \\
\hline $\mathrm{Ags} 02$ & $228 \pm 78 \mathrm{ad}$ & $295 \pm 170 b d$ & $524 \pm 247 b d$ & $7860 \pm 3711 b d$ \\
\hline Ags03 & $265 \pm 81 \mathrm{ad}$ & $390 \pm 170 b d$ & $655 \pm 251 \mathrm{bd}$ & $9832 \pm 3760 b d$ \\
\hline Dur01 & $165 \pm 72 b d$ & $219 \pm 77 b d$ & $375 \pm 144 c d$ & $5620 \pm 2163 \mathrm{~cd}$ \\
\hline Hgo01 & $193 \pm 5 \mathrm{ad}$ & $490 \pm 07 b c$ & $683 \pm 12 b d$ & $10243 \pm 176 b d$ \\
\hline Mich01 & $186 \pm 2 \mathrm{ad}$ & $479 \pm 3 b d$ & $665 \pm 5 b d$ & $9973 \pm 71 b d$ \\
\hline Mich02 & $228 \pm 8 \mathrm{ad}$ & $192 \pm 17 \mathrm{~cd}$ & $420 \pm 21 b d$ & $6299 \pm 319 b d$ \\
\hline Nay02 & $459 \pm 194 \mathrm{ab}$ & $920 \pm 163 a$ & $1379 \pm 95 a$ & $20693 \pm 1425 a$ \\
\hline Oax01 & $108 \pm 8 \mathrm{~cd}$ & $93 \pm 8 \mathrm{~cd}$ & $201 \pm 16 c d$ & $3023 \pm 237 \mathrm{~cd}$ \\
\hline Oax03 & $69 \pm 30 \mathrm{~cd}$ & $92 \pm 5 \mathrm{~cd}$ & $162 \pm 27 \mathrm{~cd}$ & $2425 \pm 399 \mathrm{~cd}$ \\
\hline Oax05 & $134 \pm 20 \mathrm{~cd}$ & $180 \pm 164 \mathrm{~cd}$ & $313 \pm 175 c d$ & $4701 \pm 2630 \mathrm{~cd}$ \\
\hline Oax07 & $89 \pm 12 c d$ & $136 \pm 61 \mathrm{~cd}$ & $225 \pm 60 \mathrm{~cd}$ & $3376 \pm 893 \mathrm{~cd}$ \\
\hline Oax 10 & $82 \pm 25 \mathrm{~cd}$ & $120 \pm 18 \mathrm{~cd}$ & $203 \pm 20 \mathrm{~cd}$ & $3039 \pm 300 \mathrm{~cd}$ \\
\hline Pue01 & $64 \pm 6 c d$ & $70 \pm 13 d$ & $135 \pm 19 d$ & $2023 \pm 285 d$ \\
\hline Pue04 & $80 \pm 2 \mathrm{~cd}$ & $121 \pm 4 \mathrm{~cd}$ & $202 \pm 5 \mathrm{~cd}$ & $3025 \pm 72 \mathrm{~cd}$ \\
\hline Qro02 & $165 \pm 84 b d$ & $285 \pm 94 b d$ & $450 \pm 178 b d$ & $6746 \pm 2671 \mathrm{bd}$ \\
\hline Qro04 & $470 \pm 246 a$ & $618 \pm 310 a b$ & $1088 \pm 556 a b$ & $16325 \pm 8341 \mathrm{ab}$ \\
\hline Qro06 & $126 \pm 35 \mathrm{~cd}$ & $190 \pm 88 \mathrm{~cd}$ & $316 \pm 122 \mathrm{~cd}$ & $4739 \pm 1828 \mathrm{~cd}$ \\
\hline Qro08 & $89 \pm 4 c d$ & $103 \pm 4 \mathrm{~cd}$ & $192 \pm 8 \mathrm{~cd}$ & $2877 \pm 122 \mathrm{~cd}$ \\
\hline Qro11 & $86 \pm 20 \mathrm{~cd}$ & $111 \pm 39 c d$ & $197 \pm 58 \mathrm{~cd}$ & $2959 \pm 877 \mathrm{~cd}$ \\
\hline Qro01 & $76 \pm 00 \mathrm{~cd}$ & $111 \pm 1 \mathrm{~cd}$ & $187 \pm 2 \mathrm{~cd}$ & $2804 \pm 24 \mathrm{~cd}$ \\
\hline Qro03 & $60 \pm 1 c d$ & $170 \pm 1 \mathrm{~cd}$ & $230 \pm 2 \mathrm{~cd}$ & $3450 \pm 24 c d$ \\
\hline Qro09 & $53 \pm 3 \mathrm{~cd}$ & $87 \pm 11 \mathrm{~cd}$ & $140 \pm 9 \mathrm{~cd}$ & $2101 \pm 133 c d$ \\
\hline Sin05 & $69 \pm 9 c d$ & $105 \pm 11 \mathrm{~cd}$ & $174 \pm 19 c d$ & $2614 \pm 289 c d$ \\
\hline Ver01 & $279 \pm 132 \mathrm{ad}$ & $190 \pm 24 \mathrm{~cd}$ & $469 \pm 156 b d$ & $7031 \pm 2337 b d$ \\
\hline Ver04 & $334 \pm 228 \mathrm{ac}$ & $471 \pm 361 b d$ & $805 \pm 589 \mathrm{ac}$ & $12079 \pm 8834 \mathrm{ac}$ \\
\hline Ver06 & $238 \pm 176 a d$ & $415 \pm 279 b d$ & $653 \pm 455 b d$ & $9798 \pm 6830 b d$ \\
\hline Ver08 & $36 \pm 00 d$ & $108 \pm 2 \mathrm{~cd}$ & $144 \pm 2 \mathrm{~cd}$ & $2161 \pm 28 \mathrm{~cd}$ \\
\hline Ver09 & $133 \pm 124 \mathrm{~cd}$ & $233 \pm 213 b d$ & $367 \pm 337 \mathrm{~cd}$ & $5504 \pm 5052 \mathrm{~cd}$ \\
\hline Ver11 & $95 \pm 75 \mathrm{~cd}$ & $174 \pm 115 \mathrm{~cd}$ & $270 \pm 190 c d$ & $4051 \pm 2847 \mathrm{~cd}$ \\
\hline Ver12 & $237 \pm 110 \mathrm{ad}$ & $323 \pm 126 b d$ & $560 \pm 230 b d$ & $8408 \pm 3445 b d$ \\
\hline Average & $162 \pm 134$ & $246 \pm 217$ & $408 \pm 337$ & $6114 \pm 5060$ \\
\hline MSD & 296 & 418 & 670 & 10047 \\
\hline
\end{tabular}

Values with different letters in each column are significantly different (Tukey, $\mathrm{p}<0.05$ ).

MSD: Minimal significant difference; C: capsaicin; D: dihydrocapsaicin; SHU: Scoville heat units. 
Postharvest handling is particularly relevant for chili peppers, as capsaicinoid content varies during ripening and decreases during storage, as these results show. Our fruit samples collected from the field were mildly to intermediately pungent (6114 SHU), like the jalapeño pepper and tabasco sauce which have a moderate hot flavor for the Mexican consumer (ChasingChilli, 2020). The field-sampled piquín fruits in the current research are suitable for low-heat dishes. In nine Mexican Cag morphotypes from the central-eastern region of Yucatán, Mexico, the average pungency (Cázares-Sánchez et al., 2005) was 70374 SHU (3584 $\mu \mathrm{g} \mathrm{g}^{-1}$ capsaicin and $1707 \mu \mathrm{g} \mathrm{g}^{-1}$ dihydro-capsaicin content). In a wild piquín landrace from Sonora, Mexico, Montoya-Ballesteros et al. (2010) reported contents of 8220 and $4240 \mu \mathrm{g} \mathrm{g}^{-1}$ capsaicin and dihydrocapsaicin, respectively, which result into a high pungency (160000 SHU). On their part, González-Zamora et al. (2013) registered 480089 SHU in piquín/chiltepín fruits from northern Mexico, a level even higher than for habanero chili (C. chinense Jacq.)

\section{Pungency changes in piquín fruits from plants grown in greenhouse conditions}

Under greenhouse conditions, the average fruit capsaicin content was 33\% higher than dihydrocapsaicin (1121 vs. 844 $\mu \mathrm{g} \mathrm{mL}{ }^{-1}$, respectively) (Table 3). Among landraces, pungency varied from low (Oax 10, $4513 \mathrm{SHU}$ ) to high levels (Ags01, 55781 SHU). Pue01 (41516 SHU) and Qro03 (40396 SHU) were other highly pungent landraces. According to the pungency scale mentioned above, most of these landraces may be classified as hot peppers, with values from 20000 to 50000 SHU. In contrast, landraces Oax07 and Oax10 had less than 5000 SHU. In any case, the greenhouse conditions greatly improved pungency in all landraces, while preserving the pungency diversity. Some landraces were of moderate pungency suitable for vast consumption, while the highly pungent landraces would be preferred by special consumers

Table 3. Capsaicinoid content in fruits of 31 piquín Mexican landraces harvested from plants grown under greenhouse conditions.

\begin{tabular}{|c|c|c|c|c|}
\hline Landrace & Capsaicin & Dihydrocapsaicin & Total capsaicinoids & Pungency \\
\hline & $\mu \mathrm{g} \mathrm{mL} L^{-1}$ & $\mu \mathrm{g} \mathrm{mL} L^{-1}$ & $\mu \mathrm{g} \mathrm{mL}^{-1}$ & SHU \\
\hline $\mathrm{Ags} 01$ & $1843 \pm 27 a$ & $1876 \pm 89 a$ & $3719 \pm 101 \mathrm{a}$ & $55781 \pm 1511 a$ \\
\hline $\mathrm{Ags} 02$ & $946 \pm 178 \mathrm{il}$ & $1098 \pm 103 b d$ & $2044 \pm 246 b e$ & $30665 \pm 3682 \mathrm{be}$ \\
\hline $\mathrm{Ags03}$ & $1733 \pm 57 \mathrm{ab}$ & $418 \pm 51 \mathrm{ei}$ & $2152 \pm 24 \mathrm{be}$ & $32278 \pm 361 \mathrm{be}$ \\
\hline Dur01 & $1524 \pm 94 b c$ & $329 \pm 23 \mathrm{gi}$ & $1852 \pm 83 \mathrm{de}$ & $27788 \pm 1240 \mathrm{de}$ \\
\hline Hgo01 & $1272 \pm 23 \mathrm{cg}$ & $350 \pm 64 \mathrm{gi}$ & $1622 \pm 69 \mathrm{de}$ & $24331 \pm 1034 \mathrm{de}$ \\
\hline Mich01 & $1485 \pm 78 b d$ & $401 \pm 126 \mathrm{fi}$ & $1887 \pm 119 \mathrm{de}$ & $28300 \pm 1781 \mathrm{de}$ \\
\hline Mich02 & $959 \pm 181 \mathrm{hl}$ & $522 \pm 22 \mathrm{dh}$ & $1481 \pm 167 \mathrm{de}$ & $22217 \pm 2500 \mathrm{de}$ \\
\hline Nay02 & $1202 \pm 31 \mathrm{di}$ & $754 \pm 24 \mathrm{ch}$ & $1957 \pm 55 \mathrm{ce}$ & $29352 \pm 823 \mathrm{ce}$ \\
\hline Oax01 & $1206 \pm 167 \mathrm{di}$ & $811 \pm 686 \mathrm{bg}$ & $2017 \pm 840$ be & $30261 \pm 1259 \mathrm{be}$ \\
\hline Oax03 & $1350 \pm 133 \mathrm{cf}$ & $389 \pm 149 \mathrm{fi}$ & $1739 \pm 275 \mathrm{de}$ & $26083 \pm 4128 \mathrm{de}$ \\
\hline Oax05 & $1043 \pm 237 \mathrm{fk}$ & $1012 \pm 319 b d$ & $2055 \pm 554 \mathrm{be}$ & $30821 \pm 8312 b e$ \\
\hline Oax07 & $141 \pm 6 \mathrm{~m}$ & $178 \pm 11 \mathrm{hi}$ & $319 \pm 17 f$ & $4784 \pm 254 f$ \\
\hline Oax 10 & $155 \pm 4 \mathrm{~m}$ & $145 \pm 38 \mathrm{i}$ & $301 \pm 34 f$ & $4513 \pm 509 f$ \\
\hline Pue01 & $1567 \pm 150 \mathrm{ac}$ & $1200 \pm 117 b c$ & $2768 \pm 267 b$ & $41516 \pm 4000 b$ \\
\hline Pue04 & $1130 \pm 143 e j$ & $1017 \pm 267 b d$ & $2147 \pm 410 \mathrm{be}$ & $32200 \pm 6147 b e$ \\
\hline Qro01 & $1283 \pm 106 \mathrm{cg}$ & $955 \pm 205 \mathrm{bf}$ & $2239 \pm 311 b e$ & $33582 \pm 4671$ be \\
\hline Qro02 & $1275 \pm 18 \mathrm{cg}$ & $991 \pm 72$ be & $2267 \pm 89 b d$ & $33999 \pm 1340 \mathrm{bd}$ \\
\hline Qro03 & $1357 \pm 116 c e$ & $1336 \pm 217 \mathrm{ab}$ & $2693 \pm 101 b c$ & $40396 \pm 1516 b c$ \\
\hline Qro04 & $1175 \pm 4 \mathrm{di}$ & $1036 \pm 8 \mathrm{bd}$ & $2210 \pm 11 \mathrm{be}$ & $33155 \pm 170 \mathrm{be}$ \\
\hline Qro06 & $1277 \pm 6 \mathrm{cg}$ & $1096 \pm 3 b d$ & $2373 \pm 9 b d$ & $35595 \pm 133 b d$ \\
\hline Qro08 & $1044 \pm 4 \mathrm{ek}$ & $835 \pm 4 b g$ & $1880 \pm 8 \mathrm{de}$ & $28196 \pm 114 \mathrm{de}$ \\
\hline Qro11 & $1047 \pm 33 \mathrm{ek}$ & $1019 \pm 152 b d$ & $2066 \pm 120 \mathrm{de}$ & $30992 \pm 1801$ be \\
\hline Qro09 & $1144 \pm 141 \mathrm{ej}$ & $1047 \pm 14 \mathrm{bd}$ & $2191 \pm 155 b e$ & $32875 \pm 2321$ be \\
\hline Sin05 & $729 \pm 341$ & $1040 \pm 20 \mathrm{bd}$ & $1770 \pm 52 \mathrm{de}$ & $26547 \pm 784 \mathrm{de}$ \\
\hline Ver01 & $1082 \pm 110 \mathrm{ej}$ & $1165 \pm 72 b c$ & $2248 \pm 178 b e$ & $33715 \pm 2663 b e$ \\
\hline Ver04 & $1206 \pm 15 \mathrm{di}$ & $892 \pm 2 b g$ & $2098 \pm 17 \mathrm{be}$ & $31467 \pm 251 b e$ \\
\hline Ver06 & $768 \pm 60 \mathrm{kl}$ & $956 \pm 254 \mathrm{bf}$ & $1724 \pm 314 d e$ & $25863 \pm 4706 \mathrm{de}$ \\
\hline Ver08 & $1037 \pm 3 \mathrm{fl}$ & $836 \pm 1 b g$ & $1873 \pm 4 \mathrm{de}$ & $28093 \pm 64 \mathrm{de}$ \\
\hline Ver09 & $1002 \pm 31 \mathrm{gl}$ & $788 \pm 284 \mathrm{bg}$ & $1790 \pm 312 \mathrm{de}$ & $26845 \pm 4674 d e$ \\
\hline Ver11 & $918 \pm 5$ il & $797 \pm 6$ bg & $1715 \pm 10 \mathrm{de}$ & $25725 \pm 142 \mathrm{de}$ \\
\hline Ver12 & $857 \pm 8 \mathrm{j} 1$ & $883 \pm 5$ bg & $1740 \pm 7 \mathrm{de}$ & $26105 \pm 97 \mathrm{de}$ \\
\hline Average & $1121 \pm 370$ & $844 \pm 393$ & $1966 \pm 633$ & $29485 \pm 9488$ \\
\hline MSD & 313.5 & 576.8 & 785.3 & 11779.7 \\
\hline
\end{tabular}

Values with different letters in each column are significantly different (Tukey, $\mathrm{p}<0.05$ ).

MSD: Minimal significant difference. C: capsaicin; D: dihydrocapsaicin; SHU: Scoville heat units. 
or suitable for capsaicinoid extraction. Since environmental and handling conditions were similar for all landraces in the greenhouse, pungency variability recorded among landraces could be attributed to inherited genetic differences.

Highly pungent landraces have a large market as many consumers prefer spicy foods and the characteristic pungency and flavor of the piquín pepper in its various presentations (sauces, pickles, dried, or fresh). The pungency levels found here in the 31 studied landraces agree with the range of 22190-44035 SHU reported for nine ecotypes of wild piquín pepper collected in northeast Mexico (Moreno-Ramírez et al., 2018). In the highly pungent habanero pepper, ContrerasPadilla and Yahia (1998) observed that fruits harvested from greenhouse-grown plants outperformed fruits from openair grown plants by $72 \%$ in accumulation of total capsaicinoids (195380 vs. $113040 \mathrm{mg} \mathrm{kg}^{-1}$ dry weight, respectively). Therefore, it can be inferred that Capsicum fruits are able to synthesize more capsaicinoids under favorable conditions than outdoors. The significant effect of environmental factors on the synthesis and accumulation of capsaicinoids was also observed by Harvell and Bosland (1997) in a dihaploid population of $C$. annuum, in which the capsaicinoid content varied up to eight-fold between different environmental conditions.

\section{Pungency changes during fruit maturation}

Across the maturation stages (green, intermediate, and red) the greenhouse piquín fruits showed significant differences ( $p<0.05)$ among maturity stages and among landraces (Table 4). For most landraces, fruit pungency increased as maturity advanced from the green stage (14813 SHU) to the intermediate stage (24767 SHU), and kept increasing to the mature red stage (29485 SHU). Similarly, Díaz et al. (2004) reported that capsaicinoids gradually accumulated through

Table 4. Pungency in piquín pepper fruits of 31 Mexican landraces harvested at three maturity stages when grown under greenhouse conditions.

\begin{tabular}{|c|c|c|c|}
\hline Landrace & Green & Intermediate & Red \\
\hline & SHU & SHU & SHU \\
\hline Ags01 & $14894 \pm 1061 \mathrm{gkC}$ & $23748 \pm 3585 \mathrm{eiB}$ & $55781 \pm 1511 \mathrm{a}$ \\
\hline $\mathrm{Ags} 02$ & $17161 \pm 1175 \mathrm{fiC}$ & $21350 \pm 912 \mathrm{gkB}$ & $30665 \pm 3682 \mathrm{be} A$ \\
\hline $\mathrm{Ags} 03$ & $22822 \pm 3455 \mathrm{acC}$ & $28896 \pm 4490 \mathrm{ceB}$ & $32278 \pm 361 \mathrm{beA}$ \\
\hline Dur01 & $18749 \pm 2375 \mathrm{dgC}$ & $23000 \pm 2007 \mathrm{ejB}$ & $27788 \pm 1240$ deA \\
\hline Hgo01 & $17169 \pm 1307 \mathrm{fiC}$ & $28442 \pm 357 \mathrm{cfB}$ & $24331 \pm 1034 \mathrm{de} A$ \\
\hline Mich01 & $10091 \pm 1736 \mathrm{moC}$ & $26825 \pm 1030 \mathrm{dgB}$ & $28300 \pm 1781 \mathrm{de} A$ \\
\hline Mich02 & $7693 \pm 1054 \mathrm{oC}$ & $36646 \pm 1015 \mathrm{abB}$ & $22217 \pm 2500 \mathrm{de} A$ \\
\hline Nay02 & $22217 \pm 1957 b d C$ & $25356 \pm 622 \mathrm{dhB}$ & $29352 \pm 823 \mathrm{ceA}$ \\
\hline Oax01 & $20078 \pm 1698 \mathrm{cfC}$ & $30732 \pm 2508 \mathrm{bdB}$ & $30261 \pm 1259 \mathrm{be} A$ \\
\hline Oax03 & $13314 \pm 266 \mathrm{imC}$ & $33696 \pm 10 \mathrm{acB}$ & $26083 \pm 4128 \mathrm{de} A$ \\
\hline Oax05 & $15923 \pm 949 \mathrm{gjC}$ & $38867 \pm 1806 \mathrm{aB}$ & $30821 \pm 8312 \mathrm{beA}$ \\
\hline Oax07 & $13057 \pm 189 \mathrm{jnC}$ & $22530 \pm 2076 f j B$ & $4784 \pm 254 \mathrm{fA}$ \\
\hline Oax 10 & $17816 \pm 809 \mathrm{ehC}$ & $27144 \pm 3836 \mathrm{dgB}$ & $4513 \pm 509 \mathrm{fA}$ \\
\hline Pue01 & $7608 \pm 1025 \circ \mathrm{c}$ & $27976 \pm 2633 \mathrm{cfB}$ & $41516 \pm 4000 \mathrm{bA}$ \\
\hline Pue04 & $14212 \pm 517 \mathrm{hiC}$ & $25734 \pm 2154 \mathrm{dhB}$ & $32200 \pm 6147 \mathrm{be} A$ \\
\hline Qro01 & $15850 \pm 457 \mathrm{gjC}$ & $24710 \pm 117 \mathrm{dhB}$ & $33582 \pm 4671 \mathrm{beA}$ \\
\hline Qro02 & $14603 \pm 332 \mathrm{hkC}$ & $27513 \pm 356 \mathrm{cgB}$ & $33999 \pm 1340 \mathrm{bdA}$ \\
\hline Qro03 & $20334 \pm 1994 \mathrm{cfC}$ & $16055 \pm 86 \mathrm{klB}$ & $40396 \pm 1516 b c \mathrm{~A}$ \\
\hline Qro04 & $13185 \pm 194 \mathrm{imC}$ & $36662 \pm 605 \mathrm{abB}$ & $33155 \pm 170 \mathrm{beA}$ \\
\hline Qro06 & $7073 \pm 303 \mathrm{oC}$ & $24772 \pm 3506 \mathrm{dhB}$ & $35595 \pm 133$ bdA \\
\hline Qro08 & $21511 \pm 2429 c e C$ & $22218 \pm 1446 \mathrm{fkB}$ & $28196 \pm 114 \mathrm{deA}$ \\
\hline Qro09 & $26107 \pm 706 \mathrm{abC}$ & $25006 \pm 157 \mathrm{dhB}$ & $32875 \pm 2321 \mathrm{beA}$ \\
\hline Qro11 & $14450 \pm 955 \mathrm{hkC}$ & $25070 \pm 99 \mathrm{dhB}$ & $30992 \pm 1801 \mathrm{beA}$ \\
\hline $\operatorname{Sin} 05$ & $11081 \pm 103 \mathrm{koC}$ & $26598 \pm 397 \mathrm{dgB}$ & $26547 \pm 784 \mathrm{deA}$ \\
\hline Ver01 & $9787 \pm 391 \mathrm{moC}$ & $19819 \pm 1157 \mathrm{hkB}$ & $33715 \pm 2663 \mathrm{beA}$ \\
\hline Ver04 & $8861 \pm 224 \mathrm{oC}$ & $15981 \pm 894 \mathrm{klB}$ & $31467 \pm 251 \mathrm{beA}$ \\
\hline Ver06 & $10236 \pm 670 \circ \mathrm{C}$ & $15990 \pm 620 \mathrm{klB}$ & $25863 \pm 4706 \mathrm{de} A$ \\
\hline Ver08 & $8817 \pm 186 \circ \mathrm{oC}$ & $20068 \pm 1656 \mathrm{hkB}$ & $28093 \pm 64 \mathrm{deA}$ \\
\hline Ver09 & $9083 \pm 78$ noC & $18120 \pm 1712 \mathrm{ikB}$ & $26845 \pm 4674 \mathrm{de} A$ \\
\hline Ver11 & $26714 \pm 194 \mathrm{aC}$ & $16782 \pm 668 \mathrm{jlB}$ & $25725 \pm 142$ deA \\
\hline Ver12 & $8702 \pm 501 \mathrm{oC}$ & $11450 \pm 3618 \mathrm{~B}$ & $26105 \pm 97 \mathrm{deA}$ \\
\hline Average & $14813 \pm 5584$ & $24767 \pm 6580$ & $29485 \pm 9487$ \\
\hline MSD & 4026 & 6305 & 11780 \\
\hline
\end{tabular}

Values with different lowercase letters in each column are significantly different $(\mathrm{p}<0.05)$. Values with different uppercase letters in each row are significantly different $(\mathrm{p}<0.05)$. 
fruit development until reaching the highest content at the end of ripening. However, some piquín landraces behaved differently. Figure 2 illustrates the behavior of three contrasting landraces through fruit maturation. Landraces Oax 10 and Mich02 increased in pungency from the green stage to the intermediate stage, and thereafter decreased sharply when reaching the red stage. However, landrace Ags01 followed the average trend: the fruit maintained an increasing pungency from the green stage to the red stage when it reached the highest pungency.

It has been reported that capsaicinoid concentration during fruit development starts a few days after flowering, followed by linear increments for the next 30 to $50 \mathrm{~d}$ to then decrease until harvest (Iwai et al., 1979). According to Estrada et al. (2000), the final upsurge until the red stage results from increased rate of capsaicin synthesis and decreased degradation rate due to diminished activity of oxidating isoenzymes. That is, capsaicinoid content in chili peppers depends on the balance between simultaneous synthesis and degradation during fruit ripening. The decreasing capsaicinoid content that occurs with fruit maturity in some genotypes has been associated to the activity of peroxidase enzymes (Bernal et al., 1993; Contreras-Padilla and Yahia, 1998).

\section{Fruit size and shape}

The piquín plants grown in greenhouse produced larger fruits than those collected in the field or purchased in markets. Greenhouse fruits were $47 \%$ longer, $43 \%$ wider, and $100 \%$ heavier than fruits collected from field-grown plants. Therefore, piquín landraces can produce larger fruits and can potentially yield more fruit when grown under greenhouse protected conditions compared to field-grown plants. Since at this experimental site there were small differences in temperature between the greenhouse and the open-air conditions, we postulate that the larger and more pungent fruits produced in the greenhouse can be attributed to a better watering and fertilization given by the drip irrigation system which provided plants with nutrient solution 9 times a day. Nonetheless, these environmental differences did not produce significant changes on the fruit roundness index (Table 5).

Among field-collected samples, fruit length ranged from 2.9 to $17.3 \mathrm{~mm}$, while the greenhouse fruits ranged from 6 to $37 \mathrm{~mm}$. The longest field-collected fruits were produced by landraces Oax10, Dur01, and Mich02, with 15.9, 17.0, and $17.3 \mathrm{~mm}$, respectively. Landraces Mich02, Nay02, and Oax10 produced the longest fruits in the greenhouse with 21.1, 21.5, and $37 \mathrm{~mm}$, respectively. Most field-collected fruits weighed less than $270 \mathrm{mg}$, except for Mich02 fruits that registered $540 \mathrm{mg}$. Similar variations in fruit size were reported by Narez-Jiménez et al. (2014) for wild chili landraces collected in the Mexican state of Tabasco. The results hint that Mich02 landrace may not belong to the Cag species because field and greenhouse fruits markedly exceeded the average size of piquín fruits (Figure 3). Only three landraces (Qro03, Qro10, and Ver08) produced fruits of similar size and weight at field locations and greenhouse conditions.

Figure 2. Pungency levels at three fruit maturation stages (green, intermediate, and red) in three contrasting piquín pepper landraces and in the average of 31 landraces.

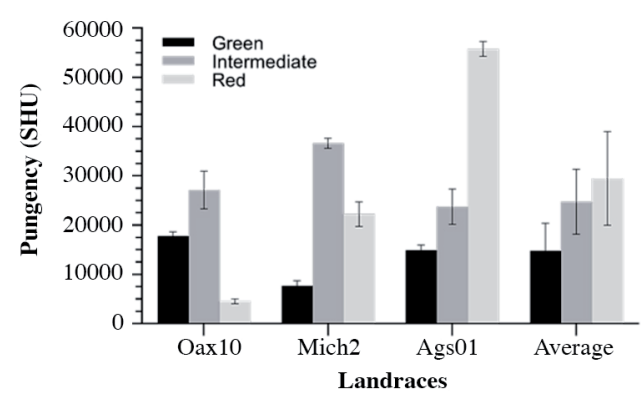

Table 5. Comparison of fruit size traits of 31 piquín pepper Mexican landraces sampled from two contrasting environments: field locations $v s$. harvested in the greenhouse $(n=124)$.

\begin{tabular}{lcccc}
\hline Environment & Length & Width & $\begin{array}{c}\text { Roundness index } \\
\text { (length/width) }\end{array}$ & Dry weight \\
\hline Greenhouse & $\mathrm{mm}$ & $\mathrm{mm}$ & & $\mathrm{g}$ \\
Field & $14.4 \pm 2.5 \mathrm{a}$ & $6.7 \pm 2.0 \mathrm{a}$ & $2.5 \pm 0.6 \mathrm{a}$ & $0.24 \pm 0.1 \mathrm{a}$ \\
\hline
\end{tabular}

Averages \pm standard deviations. Means with different letters are significantly different (Tukey, $\mathrm{p}<0.05)$. 
Figure 3. Grouping of 31 piquín pepper landraces sampled in two contrasting environments, 31 field locations vs. greenhouse grown plants, according to the cluster analysis based on fruit size and quality traits.

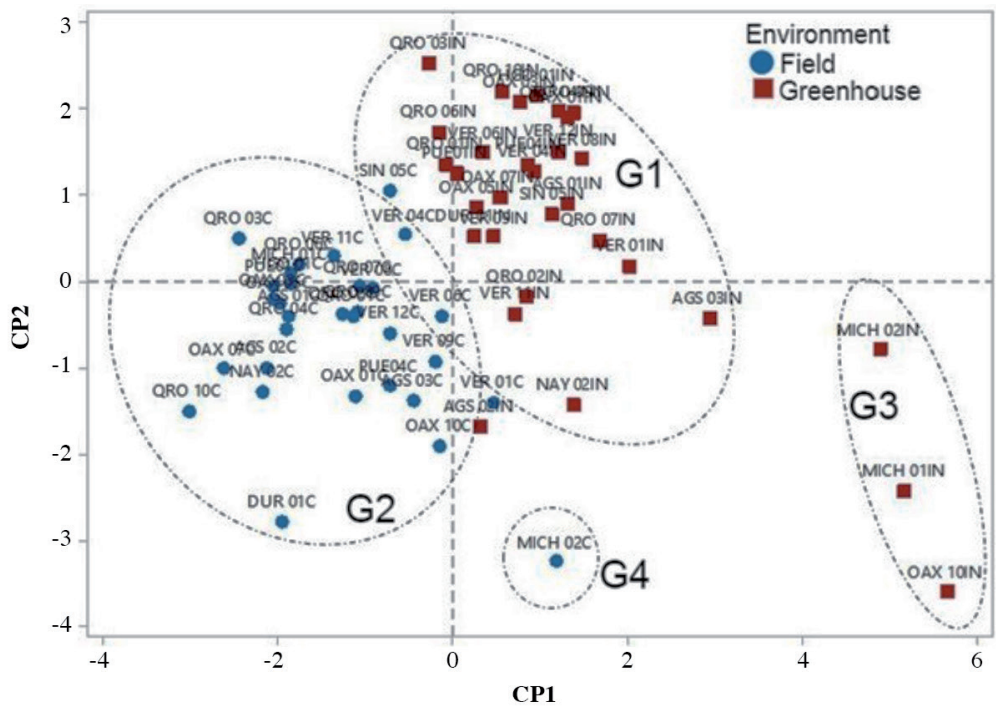

\section{Fruit quality}

Titratable acidity (TA). In most landraces, greenhouse fruits had a higher TA $(0.23 \%$ to $0.38 \%)$ than field-harvested fruits $(0.21 \%$ to $0.28 \%)$. Landraces Sin05 and Ver11 were less affected by the different growing conditions, as TA was similar in both environments. The changes recorded between environments for most landraces might be related to postharvest fruit handling. The greenhouse-collected fruits were analyzed immediately after harvest. In contrast, field-collected fruits had heterogeneous and unknown storage and handling conditions. In all cases, however, TA decreased as the fruit ripens, probably because in Capsicum the organic acids are used up for respiration (Durán-Acevedo et al., 2014). On the other hand, $\mathrm{pH}$ values varied much less than TA, since field-collected samples ranged from 4.8 to 6.4 while greenhouse-grown fruits varied between 5.1 to 5.6 .

Total soluble solids (TSS). Most landraces showed higher TSS values in fruits sampled at field locations than in greenhouse fruits. These differences might depend on the fruit maturity stage: field-collected samples were older and drier, and had a higher concentration of soluble solids than the freshly ripened fruits harvested at the greenhouse. Fieldsampled landraces Qro10 and Dur01 had 3.01 and 3.02 ${ }^{\circ}$ Brix, respectively, which were the highest recorded values. However, in the greenhouse, the highest TSS values barely exceeded 2.0 ${ }^{\circ}$ Brix by landraces Dur01, Nay02, and Qro01. TSS content increases with fruit maturation due to starch degradation (Nuez-Viñals et al., 1996). In wild piquín landraces from Nuevo León, Mexico, values ranged from 1.3 to $2.8^{\circ}$ Brix (Flores-González et al., 2018), similar to the range found in our study.

Maturity index (MI). Maturity index (TSS/TA ratio) for field-collected fruits was higher than for greenhouse-grown fruits. It indicates the balance between fruit sweetness and acidity, and it is often used as flavor quality criteria for fruits. Although MI by itself does not control fruit flavor, it allows analysis of postharvest fruit evolution. This index reveals the hydrolytic degradation of starch and pectins that increase the sweet taste (Nuez-Viñals et al., 1996), due to an increase in soluble solids and sugars associated with the decrease in acid content during ripening. Thus, the longer postharvest and storage handling should have allowed a higher concentration of soluble sugars to produce a sweeter taste.

\section{Cluster analysis for fruit size and quality}

The 31 landraces observed in two environmental conditions were classified into four differentiated groups. Group 1, located between quadrants I and II, included most greenhouse fruits. Group 2, containing most field-collected fruits, was located between quadrants II and III. Group 3 located in quadrant IV, farther from the center and contained only three 
landraces (Mich01, Mich02, and Oax10) that under greenhouse conditions produced larger fruits than on the open field. Finally, field-collected fruits of landrace Mich02 formed Group 4, because they were unusually larger and wider than other landraces. Thus, landrace Mich02 as collected from the field, may not be a piquín pepper, although it is named and sold as such. These results confirm the strong effect that favorable growth conditions under a greenhouse combined with appropriate postharvest handling have on fruit size and quality; fruits harvested in the greenhouse are larger and heavier than those collected in the field and taste more acidic (higher TA).

\section{CONCLUSIONS}

Fresh greenhouse-harvested piquín pepper fruits contain almost five times more capsaicin and dihydrocapsaicin than fruits collected from the field, which were stored and handled in unknown ways. Capsaicin content in field-collected fruits was lower than dihydrocapsaicin content, contrary to recorded capsaicinoids content in recently greenhouse-harvested fruits. After harvest, capsaicin is degraded faster than dihydrocapsaicin. During fruit ripening of greenhouse harvested fruits, $70 \%$ of the piquín landraces increased their pungency during the whole maturity stages, so they reached the highest level at the mature red stage. Only in few landraces fruit pungency decreased in their maturation from the intermediate stage to the red stage.

In most piquín landraces, greenhouse harvested fruits were longer, wider, and heavier than fruits sampled from the 31 field locations. For example, the landrace Oax10 doubled its fruit size in the greenhouse compared to its field-grown fruits. The quality traits ( $\mathrm{pH}$, acidity, total soluble solids, and maturity index) changed across the maturity stages for both field and greenhouse collected fruits. The landrace grouping based on fruit traits showed four well-differentiated groups, mostly due to the environmental growing conditions. Three landraces (Mich01, and Mich02, and Oax01) differed from all others both in the field collected and in greenhouse harvested fruits, so they might belong to a different species.

\section{REFERENCES}

Alonso, R.A., Zambrano, B., Quiroga, R., Rosales, M., y Ponce, P. 2012. Caracterización morfológica y molecular de la variabilidad genética del timpinchile (Capsicum annum L.var. glabriusculum sin. aviculare) en Chiapas. Quehacer Científico en Chiapas 1(13):4-18.

AOAC. 1995. Official methods of analysis. Association of Analytical Chemists (AOAC), Washington D.C., USA.

Bañuelos, N., Salido, P.L., y Gardea, A. 2008. Etnobotánica del chiltepín. Pequeño gran señor en la cultura de los sonorenses. Estudios Sociales 16(32):177-205.

Barchenger, D.W., and Bosland, P.W. 2016. Exogenous applications of capsaicin inhibit seed germination of Capsicum annuum. Scientia Horticulturae 203:29-31. doi:10.1016/j.scienta.2016.03.009.

Bernal,A.M., Calderón, A.A., Pedreño, M.A., Muñoz, R., Barceló, A.R., and Merino, F. 1993. Capsaicin oxidation by peroxidase from Capsicum annuum (var. annuum) fruits. Journal of Agricultural and Food Chemistry 41:1041-1044.

Cano-Vázquez, A., López-Peralta, M., Zavaleta-Mancera, H.A., Cruz-Huerta, N., Ramírez-Ramírez, I., Gardea-Béjar, A.A., et al. 2015. Variación en grados de latencia en semillas entre colectas de chile piquín (Capsicum annuum var. glabriusculum). Botanical Sciences 93:175-184. doi:10.17129/botsci.138.

Cázares-Sánchez, E., Rodríguez-González, M.T., Soto-Hernández, R., Chávez-Servia, J.L., Castillo-González, F., and RamírezVallejo, P. 2005. Capsaicinoids and preference of use in different morphotypes of chili peppers (Capsicum annuum L.) of East-Central Yucatán. Agrociencia 39:627-638.

Chasing-Chilli. 2020. Official Scoville scale. 2020. Scoville scale: Ultimate list of pepper's \& their Scoville heat units. Available at https://chasingchilli.com.au/scoville-scale/ (accessed in June 2021).

Contreras-Padilla, M., and Yahia, E.M. 1998. Changes in capsaicinoids during development, maturation, and senescence of chile peppers and relation with peroxidase activity. Journal of Agricultural and Food Chemistry 46:2075-2079.

de Aguiar, A.C., Coutinho, J.P., Barbero, G.F., Godoy, H.T., and Martínez, J. 2016. Comparative study of capsaicinoid composition in Capsicum peppers grown in Brazil. International Journal of Food Properties 19:1292-1302. doi:10.1080/10942912.2015.1072210.

Díaz, J., Pomar, F., Bernal, A., and Merino, F. 2004. Peroxidases and the metabolism of capsaicin in Capsicum annuum L. Phytochemistry Reviews 3:141-157. doi:10.1023/B:PHYT.0000047801.41574.6e.

Durán-Acevedo, C.M., Gualdron-Guerrero, O.E., y Hernández-Ordoñez, M. 2014. Nariz electrónica para determinar el índice de madurez del tomate de árbol (Cyphomandra betacea Sendt). Ingeniería Investigación y Tecnología 15(3):351-362.

Estrada, B., Bernal, M.A., Díaz, J., Pomar, F., and Merino, F.C. 2000. Fruit development in Capsicum annuum: changes in capsaicin, lignin, free phenolics, and peroxidase patterns. Journal of Agricultural and Food Chemistry 48:6234-6239. 
Feldmann, F., and Rutikanga, A. 2021. Phenological growth stages and BBCH-identification keys of Chilli (Capsicum annuum L., Capsicum chinense Jacq., Capsicum baccatum L.) Journal of Plant Disease and Protection 128:549-555. doi:10.1007/s41348-020-00395-x.

Flores-González, P., Franco-Bañuelos, A., Hernández-Martínez, J., Moreno-Limón, S., Hernández-Pineiro, J.L., y Pinedo-Espinoza, J.M. 2018. Evaluación fisicoquímica y capacidad antioxidante de chiltepín silvestre de Nuevo León, México. Investigación y Desarrollo en Ciencia y Tecnología de Alimentos 3:529-534. http://www.fcb.uanl.mx/IDCyTA/files/volume3/4/9/88.pdf.

González-Zamora, A., Sierra-Campos E., Luna-Ortega, J.G., Pérez-Morales, R., Rodríguez, O.J.C., and GarcíaHernández, J.L. 2013. Characterization of different Capsicum varieties by evaluation of their capsaicinoids content by high performance liquid chromatography, determination of pungency and effect of high temperature. Molecules 18:13471-13486. doi:10.3390/molecules181113471.

González-Zamora, A., Sierra-Campos, E., Pérez-Morales, R., Vázquez-Vázquez, C., Gallegos-Robles, M.A., López-Martínez, J.D., et al. 2015. Measurement of capsaicinoids in chiltepin hot pepper: A comparison study between spectrophotometric method and high performance liquid chromatography analysis. Journal of Chemistry 2015:709150. doi:10.1155/2015/709150.

Harvell, K.P., and Bosland, P.W. 1997. The environment produces a significant effect on pungency of chiles. HortScience 32:1292. doi:10.21273/HORTSCI.32.7.1292.

Hernández-Verdugo, S.,Luna-Reyes, R., and Oyama, K. 2001. Genetic structure and differentiation of wild and domesticated populations of Capsicum annuum (Solanaceae) from México. Plant Systematics and Evolution 226:129-142. doi:10.1007/s006060170061.

Hernández-Verdugo, S., Porras, F., Pacheco-Olvera, A., López-España, R.G., Villarreal-Romero, M., Parra-Terraza, S., et al. 2012. Caracterización y variación ecogeográfica de poblaciones de chile (Capsicum annuum var. glabriusculum) silvestre del noroeste de México. Polibotánica 33:175-191. https://www.polibotanica.mx/ojs/index.php/polibotanica/article/view/860/1078.

IPGRI, AVRDC, CATIE. 1995. Descriptors for Capsicum (Capsicum spp.) International Plant Genetic Resources Institute (IPGRI), Rome, Italy; Asian for Vegetable Research and Development Center (AVRDC), Taipei, Taiwan; Centro Agronómico Tropical de Investigación y Enseñanza (CATIE), Turrialba, Costa Rica.

Iwai, K., Suzuki, T., and Fujiwake, H. 1979. Formation and accumulation of pungent principle of hot pepper fruits, capsaicin and its analogues, in Capsicum aпnиит var. annuит cv. Karayatsubusa at different growth stages after flowering. Agricultural and Biological Chemistry 43(12):2493-2498.

Martínez, L., Cilia, I., Beltrán, J.A., and Roncales, P. 2006. Effect of Capsicum annuum (red sweet and cayenne) and Piper nigrum (black and white) pepper powders on the shelf life of fresh pork sausages packaged in modified atmosphere. Journal of Food Science 71:48-53. doi:10.1111/j.1365-2621.2006.tb12405.x.

Montoya-Ballesteros, L.C., Gardea-Béjar, A., Ayala-Chávez, G.M., Martínez-Núñez, Y.Y., y Robles-Ozuna, L.E. 2010. Capsaicinoides y color en chiltepín (Capsicum annuum var. aviculare). Efecto del proceso sobre salsas y encurtidos. Revista Mexicana de Ingeniería Química 9(2):197-207. http://rmiq.org/ojs311/index.php/rmiq.

Moreno-Ramírez, Y.R., Martínez-Ávila, G.G., González-Hernández, V.A., Castro-López, C., and Torres-Castillo, J.A. 2018. Free radical-scavenging capacities, phenolics and capsaicinoids in wild Piquin chili (Capsicum annuum var. glabriusculum). Molecules 23:2655. doi:10.3390/molecules23102655.

Murillo-Amador, A.B., Rueda-Puente, O.E., Troyo-Diéguez, E., Córdoba-Matson, M.V., Hernández-Montiel, L.G., and Nieto-Garibay, A. 2015. Baseline study of morphometric traits of wild Capsicum annuum growing near two biosphere reserves in the Peninsula of Baja California for future conservation management. BMC Plant Biology 15:1-18. doi:10.1186/s12870-015-0505-6.

Narez-Jiménez, C.A., de-la-Cruz-Lázaro, E., Gómez-Vázquez, A., Castañón-Nájera, G., Cruz-Hernández, A., y MárquezQuiroz, C. 2014. La diversidad morfológica in situ de chiles silvestres (Capsicum spp.) de Tabasco, México. Revista Fitotecnia Mexicana 37(3):209-215.

Nuez-Viñals, F., Gil-Ortega, R., y Costa-Garcia, J. 1996. El cultivo de pimientos, chiles y ajíes. Mundi Prensa Libros, México.

Quintero Barrera, L. 2000. Evaluación de la diversidad genética del género Capsicum sp. presente en los Departamentos de Vaupés, Guainía y Putumayo por medio de isoenzimas. Acta Biológica Colombiana 5(1):81-82. (Abstract).

Rodríguez-Maturino, A., Valenzuela-Solorio, A., Troncoso-Rojas, R., González-Mendoza, D., Grimaldo-Juárez, O., Aviles-Marín, M., et al. 2012. Antioxidant activity and bioactive compounds of Chiltepin (Capsicum annuum var. glabriusculum) and Habanero (Capsicum chinense): A comparative study. Journal of Medicinal Plants Research 6:1758-1763. doi:10.5897/JMPR11.1576.

Sandoval-Rangel, A., Tapia, A., Cabrera-De la Fuente, M., González, J., y Benavides-Mendoza, A. 2018. Edad, beneficio y ácido giberélico afectan la germinación y producción de planta de chile piquín. Revista Mexicana de Ciencias Agrícolas 20:4199-4209. doi:10.29312/remexca.v0i20.990.

Steiner, A.A. 1961. A universal method for preparing nutrient solutions of a certain desired composition. Plant and Soil 15:134154. doi:10.1007/BF01347224.

Villalón, H., Medina, T., and Ramírez, M. 2013. Quality factors of wild chili seed (Capsicum annuum L. var. glabriusculum). Revista Mexicana de Ciencias Forestales 4:182-187.

Wall, M.M., Waddell, C.A., and Bosland, P.W. 2001. Variation in $\beta$-carotene and total carotenoid content in fruits of Capsicum. HortScience 36(4):746-749. 